\title{
MS31-P01 | STRUCTURAL ANALYSIS AND IR-SPECTROSCOPY OF A NEW ANILINIUM HYDROGENSELENITE HYBRID COMPOUND: A SUBTLE STRUCTURAL PHASE TRANSITION
}

takouachet, Radhwane (Abbes Laghrour Khenchela University, Khenchela, DZA); Benali-Cherif, Rim (Abbes Laghrour Khenchela University, khenchela, DZA); Bendeif, El-Eulmi (Laboratoire de Cristallographie, Résonance Magnétique et Modélisations CRM2, Lorraine university, Nancy, FRA); Benali-Cherif, Nourredine (Bouira University, Bouira, DZA)

The hybrid compounds have been extensively studied over the last few decades for their specific properties and potential applications. These compounds display a wide range of molecular interactions network, from strong ionic and hydrogen bonding to weak van der Waals contacts. The asymmetric unit of this compound consists of two anilinium organic cations, two hydrogenselenite anions and one water molecule.

The temperature dependent structural investigation reveals that the studied compound undergoes a subtle noncentrosymmetric to centrosymmetric structural phase transition. At room temperature the crystal structure is noncentrosymmetric and is characterized by an important disorder in the organic part. By decreasing the temperature to $100 \mathrm{~K}$ the organic cation is less disordered and the rotation angle of the aromatic rings changes. As a consequence, the low temperature structure becomes centrosymmetric. The infrared spectra recorded on cooling and heating the sample in the temperature range of 300-9K support this analysis. $\mathrm{NH}_{3}$ is significantly affected by the temperature change. This effect results, when cooling the sample below $140 \mathrm{~K}$, in the appearance of a new vibrational band corresponding to the $\left(\mathrm{NH}_{3}\right)$-wagging modes at $705 \mathrm{~cm}^{-1}$. The phenomenon is completely reversible and the new vibrational band disappears upon heating the sample above $140 \mathrm{~K}$. Moreover, the $\mathrm{v}(\mathrm{C}-\mathrm{N})$-stretching and the $\left(\mathrm{NH}_{3}\right)$-scissoring modes are also affected by the temperature variation as can be seen in the spectral ranges of $1250-1400 \mathrm{~cm}^{-1}$ and $1610-1635 \mathrm{~cm}^{-1}$. 